\title{
A sono-anatomical and cadaveric study of ultrasound-guided retrolaminar block
}

\author{
Fahd Aamir ${ }^{1}$, Michael Cronin², Peter Lee', Gabriella Iohom¹, George Shorten ${ }^{1}$ \\ ${ }^{1}$ Department of Anaesthesia and Intensive Care Medicine, Cork University Hospital and University College Cork, \\ ${ }^{2}$ Department of Anatomy and Neuroscience, University College Cork, Ireland
}

\begin{abstract}
Aim: Retrolaminar block (RB) is known to confer chest wall analgesia but, its mechanism has not been established. Our primary objective was to determine if the spread of injectate following RB extends to the paravertebral space (PVS). Secondary objectives were to determine the predefined anatomical areas and nervous tissues contacted by injectate; the effect of volume on spread; and the extent to which experts can predict PVS spread based on examination of US videos of the injection. Material and methods: US-guided RB was performed on cadavers using a single injection technique of 10, $20 \mathrm{or} 30 \mathrm{ml}$ dye. Anatomical dissection was performed to identify the extent of spread of injectate to the retrolaminar, intercostal and PVS. Ultrasound recordings of the injection were independently evaluated by experts in US-guided regional anaesthesia. Results: Spread of injectate to the ipsilateral PVS was identified in $6 / 10$ dissected regions $(0 / 1,1 / 3$ and $5 / 6$ when injectate volumes of 10, 20 and $30 \mathrm{ml}$ were administered respectively). The extent of cephalad-caudad spread within the PVS varied from 1 to 3 levels. Expert interpretation of ultrasound images regarding spread to the PVS demonstrated poor correlation with dye staining observed on dissection. Conclusions: Injectate spread following RB demonstrated substantial variability. Inconsistent spread to the ipsilateral PVS may account for clinically occurring incomplete blocks. The likelihood of spread to the ipsilateral PVS was greater when a larger volume was injected. Expert evaluation of the dynamic ultrasound images obtained at injection cannot reliably predict spread to the PVS.
\end{abstract}

Keywords: regional anaesthesia; nerve block; cadaver; anatomy; ultrasound

\section{Introduction}

Paravertebral blockade (PVB) has a well-established role for providing analgesia of the thoracic cage and, in some settings, offers an effective alternative to thoracic epidural analgesia. When compared with opioid-based analgesia, PVB is associated with lesser pain scores in the postoperative period [1]. A Cochrane review concluded that PVB decreases the incidence of chronic

Received 05.12.2020 Accepted 18.03.2021

Med Ultrason

2021:0 Online first, 1-6

Corresponding author: Fahd Aamir, MB BCh BAO, FCAI

Department of Anaesthesia and Intensive Care Medicine, Cork University Hospital,

Cork, Ireland

E-mail: fahdaamir@icloud.com

Phone: +353 (21) 4922134 post-surgical pain in breast surgery patients at one year postoperatively [2]. In trauma patients, approximately $10 \%$ have rib fractures $[3,4]$ and regional anaesthesia has been advocated for pain management in thoracic trauma [5]. In a pilot study, PVB and thoracic epidural provided equivalent analgesia when performed for rib fracture management [6]. For thoracotomy pain, moderate quality evidence from a Cochrane review showed that PVB resulted in similar analgesic efficacy compared with thoracic epidural analgesia [7].

During performance of PVB, the proximity of pleura and lung tissue presents a risk of pneumothorax $(0.5 \%$ for landmark based techniques) [8,9]. Although ultrasound guidance may mitigate this risk, no consensus exists on which US-guided technique is optimal $[10,11]$. The recognized risk of pneumothorax, particularly in a setting in which positive pressure ventilation is imminent, may dissuade anaesthesiologists form performing this block. 
A more recently described technique, retrolaminar paravertebral block (RB), may offer analgesic effect equivalent to standard PVB with lesser risk of pneumothorax. It is proposed that injection of local anaesthetic directly on to the posterior aspect of the vertebral lamina results in spread or diffusion through the superior costotransverse ligament to the paravertebral space (PVS). The putative advantages of this technique are that; i) its point of injection and endpoint of needle insertion is the lamina, a well-defined bony structure which is easily visualised on ultrasound scanning, ii) it does not rely on loss of resistance tactile feedback for identification of the lamina, and iii) the point of injection can be identified using in-plane ultrasound guidance. Its effect depends on local anaesthetic spread from the point of injection, thereby avoiding contact with and the risk of direct injury to pleura and nerve tissue. Its use and clinical efficacy have been described in several case reports and in a number of small clinical trials [12-15]. The amount and quality of clinical evidence currently available is insufficient to establish its safety or the consistency of its analgesic effect.

To justify a change in practice towards more widespread use of RB, greater understanding of its mechanism of action is required. In practical terms, this means identification of the nerves blocked and the routes by which RB injectate reaches them. This requires study of the spread of injectate associated with the block. Recently, a carefully performed cadaver study demonstrated marked variability in the spread of $20 \mathrm{ml}$ of bupivacaine $0.5 \%$ with methylene blue $1 \%$ [16]. At dissection, injected dye was identified in the PVS of 5 of 8 cadavers studied, the adjacent intercostal space in 5 of 8 cadavers, the adjacent epidural space in 6 of 8 cadavers and the adjacent intervertebral foramina in 4 of 8 cadavers. It is notable that, in this relatively small number of experiments, no single neural target was consistently accessed by RB injection. [16]

The above findings prompt a further question: can performance of RB be modified to achieve consistent spread of injectate to the PVS (or another potential site of neural sensory blockade of the chest wall)? We have conducted a cadaveric study of RB to extend the data available on spread of injectate, specifically addressing to the effect of volume of injectate and to the extent to which US images of the injection can be interpreted by experts to predict spread to the PVS. Our primary objective was to determine if spread of injectate of clinically relevant volumes following RB extends to the paravertebral space. The secondary objectives were to determine the predefined anatomical areas (See Appendix) and nervous tissues contacted and overlaid by injectate, to define the effect of volumes $(10,20$ and $30 \mathrm{ml})$ on spread of injectate following RB and to determine the extent to which clinicians with expertise in US guided regional anaesthesia can predict whether injectate spreads to the PVS based on examination of US video of the injection.

\section{Materials and methods}

The study was carried out at the Department of Anatomy and Neuroscience FLAME laboratory at University College Cork (UCC), Ireland between July 2017 and July 2018. With the approval of the Head of the Department of Anatomy and Neuroscience at UCC, the designated authority for conduct of research on cadavers under the Anatomy Act Ireland, (first dissection performed $26^{\text {th }}$ Oct 2017), five cadavers were studied (Table I). All cadaver specimens were fresh frozen and were thawed to room temperature before any assessment or interventions were performed. The cadavers did not have any prior spinal or thoracic surgery and were free of spinal or thoracic cage pathology. Sonographic image capture and guided injection was carried out by an experienced sonographer (who had completed more than 400 ultrasound guided regional anaesthetic procedures over the preceding two years).

\section{Pre-injection US evaluation}

Each cadaver specimen was placed in the prone position. The initial scan identified the first rib in each hemithorax and the US probe was then gently translated in a caudad direction until the target rib was identified. The probe (L14-5W/60 Linear Probe, SonixTouch, Ultrasonix, Vancouver, Canada) was then translated medially to identify the target lamina. Once the target lamina was identified, a 'seeker' needle was inserted on the skin to mark the RB target.

Next, a pre-injection recording of the retrolaminar space of the vertebrae, cephalic and caudad to the target space was made. The probe was then moved laterally over the intercostal space, just as the rib image diverged from that of the adjacent transverse process. Imaging of the retrolaminar space cephalic and caudad to this was also made. This was done prior to all injections.

In total, ten scout scans were performed and no anatomical abnormalities were identified.

Table I. Demographics of fresh frozen cadavers

\begin{tabular}{llll}
\hline Cadaver & Sex & Age & Cause of Death \\
\hline 1 & $\mathrm{M}$ & 92 & Stroke \\
2 & $\mathrm{M}$ & 84 & Prostate Cancer \\
3 & $\mathrm{~F}$ & 86 & Heart Disease / Pneumonia \\
4 & $\mathrm{~F}$ & 64 & Breast Cancer \\
5 & $\mathrm{M}$ & 61 & Prostate Cancer \\
\hline
\end{tabular}




\section{Solution injection}

The insertion site of an $18 \mathrm{G}$ Touhy needle (B-Braun Perifix 18G Touhy Needle, Medical Ltd. Dublin 12, Ireland) was identified using US based techniques. The needle was inserted under US guidance using an in-plane technique, as described by Voscopoulos et al [3]. The US probe was placed in the sagittal paramedian (cephaladcaudad orientation) plane approximately $1 \mathrm{~cm}$ lateral to the midline spinous process of the target lamina.

Once the needle tip contacted the target lamina and the tip confirmed as deep to the para-spinous muscles, the 10,20 or $30 \mathrm{ml}$ of dye solution was injected over $10 \mathrm{sec}$ onds by an investigator (FA). The solution was composed of $0.9 \%$ saline solution of either 9-, 19- or $29 \mathrm{ml}$ volumes mixed with a $1 \mathrm{ml}$ methylene blue $1 \%$ solution (Blue Marker Aguettant, AGUETTANT LTD, Bristol, UK). Confirmation of correct needle tip position was made by identifying spread of injectate in a plane between the lamina and the adjacent paraspinous muscle.

\section{Peri/Post-injection US evaluation}

As the injection was performed and immediately afterwards, a single operator (FA) evaluated the spread of injectate in real time using live sonographic images. Vertical spread was evaluated along the laminae of the vertebral column. Horizontal spread was evaluated by US by inspecting the nearby intercostal spaces and adjacent muscle, fascial and paravertebral planes (fig 1).

\section{Dissection phase}

After the US evaluation phase and under the guidance of an experienced anatomy prosector, the subject was dissected in order to identify: Touhy needle tip position and related to volume of injectate the tissue planes marked by dye solution, nervous tissue marked by dye solution and horizontal and vertical spread of dye solution.
The Touhy needle was secured in situ. The muscles of the posterior thorax and the erector spinae muscles were dissected in layers and retracted. Once the target lamina was contacted and the overlying erector spinae muscles were dissected, the extent of dye marking was recorded. The Touhy needle contact point on the target laminae was noted and the needle then removed to enable further dissection. The cephalic and caudad erector spinae muscles were then systematically dissected to enable visualization of dye in the retrolaminar space.

The intercostal muscles were then dissected in layers to attempt to visualize spread within the muscle planes. In the later four dissections the intercostal nerves were isolated and dissected off the rib, then carefully dissected back to their origin as spinal nerves in the paravertebral space. The dorsal ramus of the spinal nerve was also identified at this point as it separated from the spinal nerve. To facilitate dissection and identification of neural structures, the ribs were sectioned and removed $1 \mathrm{~cm}$ distal to costo-transverse ligament. After the PVS had been examined for dye marking, the anterior-lateral vertebral space was opened to identify dye spread to this area. A fibreoptic scope was utilized (Ambu aScope, Ambu A/S, Ballerup, Denmark) to examine the intervertebral foramina for dye spread once the paravertebral space was dissected to avoid disruption of surrounding structures and affecting dye identification.

The second cadaver we dissected manifested early decomposition when dissection was performed. Presumably as a result, the dye staining visualised during its dissection was minimal and a definite assessment of dye spread in this specimen was difficult to make. After this we modified our dissection method, dissecting the intercostal and paravertebral spaces first to assess our primary

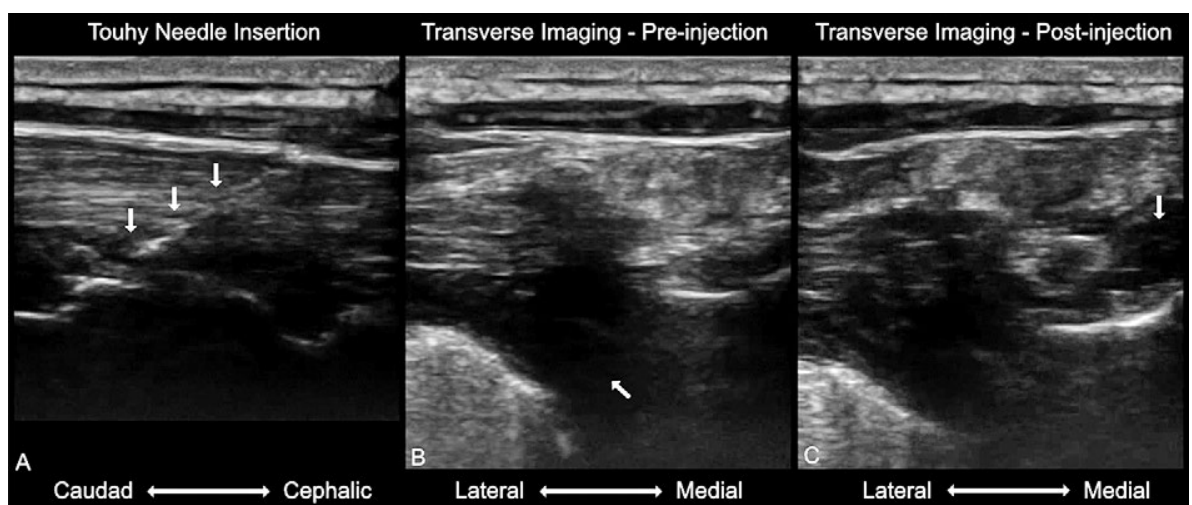

Fig 1. Ultrasound image captures. (A) Touhy needle insertion along paramedian spine in longitudinal plane (White Arrows). The needle is passing through erector spinae muscles and the tip of the needle is in contact with the Lamina to allow injection beneath the erector spinae muscles. (B) Pre-injection Image. Ultrasound is re-orientated in transverse plane to visualise Paravertebral Space (White Arrow) while needle remains in contact with lamina. Pleura is visualised underneath paravertebral space. (C) Post-injection Image. The Retro-laminar Space expands with injectate above the Lamina (White Arrow). 
objective before dissecting the other tissues to determine dye spread to other tissues.

\section{Independent assessment of US recordings}

Ultrasound images of spread of injectate was evaluated in real time by a single investigator who performed the blocks and subsequently reviewed, based on video recordings, by two independent clinicians experienced in US-guided PNB. The independent assessors examined the US data to determine if injectate spread to the PVB space could be visualised during or after injection.

\section{Statistical analysis}

Descriptive statistics was used to quantify the spread of dye to predefined anatomical areas and nervous tissues. Fisher's Exact test was performed to assess the proportionate agreement between the expert assessors. A p-value of 0.05 was considered as the threshold for statistical significance.

\section{Results}

\section{Dissection findings}

A total of 10 dissections (one on each side) were performed on five fresh frozen cadavers. In total, $10 \mathrm{RB}$ and subsequent dissections were performed: $10 \mathrm{ml}$ injection was administered for one procedure, $20 \mathrm{ml}$ injection was administered for three procedures and $30 \mathrm{ml}$ injection was administered for six procedures (Table II).

Dye was not visualised superficial to the erector spinae muscle or along the needle track superficial to the erector spinae muscles in any cadaver. Only when the erector spinae were reflected (in each case) and the retrolaminar space visualised underneath it was dye visible for the first time showing the erector spinae muscle acts as the superficial barrier to the retrolaminar space. All ten dissections demonstrated dye in the retrolaminar space deep to the erector spinae muscle. This dye spread varied from 3 levels to 8 levels and was not dose dependent. Only in two injections with 20 and $30 \mathrm{ml}$ the dye spread was visualised to the intercostal space. This was limited to one level only for each injection and present only in a single cadaver specimen. No dye staining was seen in proximity to the anterior-lateral vertebrae, pleura or at the intervertebral foramina. Medial spread of dye to the epidural space was not visible in any region.

Dye was evident in the PVS in six of the ten regions dissected. Of these, dye was visible from posteriorly in two and from anteriorly (when ribs were sectioned to permit viewing from the anterior aspect) in four. The presence of dye in the PVS appeared to be associated with the volume injected: not present in the single region to which $10 \mathrm{ml}$ injected, present in 1 of 3 regions to which $20 \mathrm{ml}$ was injected and in 5 of 6 regions to which $30 \mathrm{ml}$ injected. The extent of the superior-inferior spread of dye within the paravertebral space varied form 1-3 levels in these six regions. In cadaver number two which demonstrated partial decomposition on dissection, minimal dye staining was visible of any tissues.

During the dissections, the intercostal nerves were dissected carefully off the ribs to their origin from the intervertebral foramina into the PVS. Staining of the intercostal nerve adjacent to the lamina at which the injection was made was evident in all four regions in which these nerves were dissected individually (fig 2).

\section{Interpretation of US findings}

For the first two specimens a series of before and after injection still image recordings were available for assessment, whereas video recordings of the injections were used for the other eight specimens.

The proportionate agreement between "real time" expert assessment of the ultrasound image during and

Table II. Injectate and spread pattern of retrolaminar injection in 5 fresh frozen cadavers

\begin{tabular}{|c|c|c|c|c|c|c|c|c|c|}
\hline Cadaver & Side & $\begin{array}{l}\text { Target } \\
\text { laminae }\end{array}$ & $\begin{array}{l}\text { Depth of } \\
\text { laminae } \\
(\mathrm{cm})\end{array}$ & $\begin{array}{l}\text { Volume } \\
\text { injected } \\
(\mathrm{ml})\end{array}$ & $\begin{array}{l}\text { PV } \\
\text { spread }\end{array}$ & $\begin{array}{l}\text { Inter- } \\
\text { costal } \\
\text { space } \\
\end{array}$ & $\begin{array}{l}\text { Retro- } \\
\text { laminar } \\
\text { space }\end{array}$ & $\begin{array}{l}\text { Ant-Lat } \\
\text { foramina }\end{array}$ & $\begin{array}{l}\text { Inter- } \\
\text { vertebral } \\
\text { foramina }\end{array}$ \\
\hline \multirow[t]{2}{*}{1} & Right & & & 20 & 3 levels & 1 level & 6 levels & Nil & Nil \\
\hline & Left & & & 30 & 2 levels & 1 level & 5 levels & Nil & Nil \\
\hline \multirow[t]{2}{*}{2} & Right & T6 & 1.5 & 30 & Nil & Nil & 5 levels & Nil & Nil \\
\hline & Left & $\mathrm{T} 6$ & 1.5 & 10 & Nil & Nil & 6 levels & Nil & Nil \\
\hline \multirow[t]{2}{*}{3} & Right & T5 & 1.5 & 20 & Nil & Nil & 8 levels & Nil & Nil \\
\hline & Left & $\mathrm{T} 4$ & 2.5 & 20 & Nil & Nil & 7 levels & Nil & Nil \\
\hline \multirow[t]{2}{*}{4} & Right & T6 & 1.5 & 30 & 3 levels & Nil & 7 levels & Nil & Nil \\
\hline & Left & T6 & 1.5 & 30 & 3 levels & Nil & 5 levels & Nil & Nil \\
\hline \multirow[t]{2}{*}{5} & Right & $\mathrm{T} 7$ & 3.5 & 30 & 2 levels & Nil & 3 levels & Nil & Nil \\
\hline & Left & $\mathrm{T} 8$ & 3.5 & 30 & 1 level & Nil & 3 levels & Nil & Nil \\
\hline
\end{tabular}

PV paravertebral, T thoracic 
after injection and dissection-based detection determination of whether injectate entered the PVS was $0.75(6 / 8$, $\mathrm{p}=0.49$ ).

The proportionate agreement (on whether injectate entered the PVS) between the two expert assessors who reviewed video of the ultrasound images during and after injection was $0.375(3 / 8, \mathrm{p}=1)$. Table III details the marked variability in the interpretation of PVS entry by the injected solution between all three assessors and the subsequent dissection findings.

\section{Discussion}

Our findings indicate that spread of injectate following $\mathrm{RB}$, including to the PVS is variable. This variation is present even when the block is performed by a single operator using a standardised technique and in the absence of obvious anatomical abnormality. Injection of a greater volume (i.e. $30 \mathrm{ml}$ ) appears to increase the likelihood (dye found in PVS of 5/6 dissections) of spread to the PVS. Spread to the PVS was not reliably predicted by expert evaluation of US images acquired in real time (during the injection) or on subsequent video review.

In those injections that spread to the PVS, the extent of spread was variable even when greater volumes were injected. This PVS spread may be limited as evidenced by subtle marking of the spinal nerves in the PVS. Taken in conjunction with clinical studies [12-15] one might hypothesize that even modest spread of local anaesthetic to the PVS can result in analgesic effect.

Relative to PVB, a larger volume of solution is injected into the retrolaminar space, and this solution spreads consistently (10/10 specimens) along the retrolaminar spaces (ranging 3-8 cranio-caudad levels). This space contains the dorsal rami of the spinal nerves which conduct sensation and power to/from the erector spinae

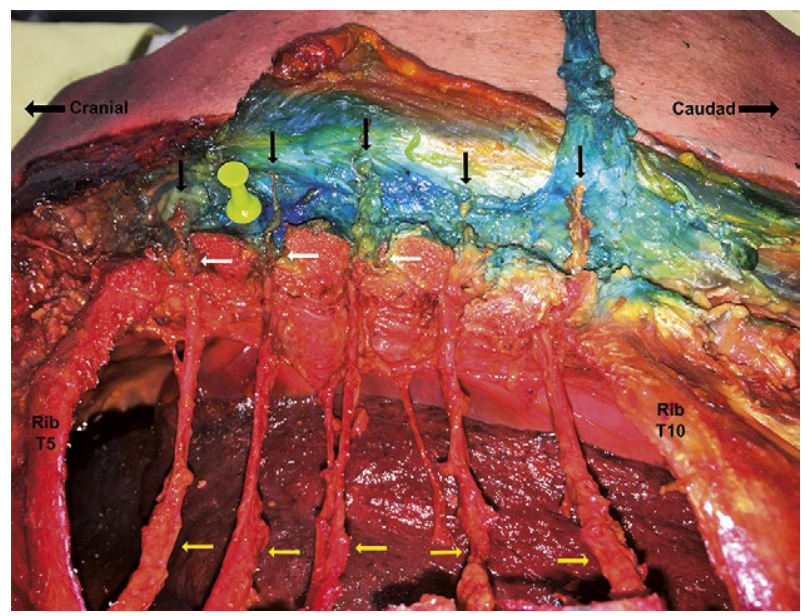

Fig 2. Left hemithorax after dissection. The Touhy needle injection point is marked by the green pin and blue dye is seen to stain the retro-laminar spaces of multiple vertebrae. The left ribs from 6 to 9 have been dissected away while the intercostal nerves (Yellow Arrows) have been spared to allow visualization of nerve structures within the Paravertebral Space. Blue dye staining of the T5, T6 and T7 spinal nerves within the Paravertebral Space is demonstrated (White Arrows). The Dorsal Rami of the spinal nerves (Black Arrows) are also visualised stained with blue dye.

muscles. This effect may account for some of the clinical benefit observed when this block is performed. For patients who have sustained rib fractures, these muscles may be in spasm and their relaxation secondary to RB may account for part of the resultant analgesic benefit.

Compared to the results obtained by Sabouri et al [16], our dye staining of the spinal nerves was more subtle, which may have been influenced by our cadaver specimens remaining prone throughout the injection and dissection phases of the study. To minimize the possibility of artefact influencing the degree of dye staining observed, cadavers were retained in the position in which

Table III. Comparison of real-time and recorded ultrasonographic interpretation change in PV space compared to cadaver dissection

\begin{tabular}{llllll}
\hline Cadaver & Side & PV Space Entered & & & Assessor Two \\
\cline { 3 - 6 } & & Real-Time & Assessor One & Dissection \\
\hline 1 & Right & No Video Data & & & \\
\multirow{2}{*}{2} & Left & No Video Data & & & No \\
& Right & No & Yes & No & No \\
3 & Left & Yes & Yes & No & No \\
& Right & No & Yes & Yes & No \\
4 & Left & No & Yes & Yes & Yes \\
& Right & Yes & Yes & No & Yes \\
5 & Left & Yes & Yes & Yes & Yes \\
\hline & Right & Yes & No & No &
\end{tabular}

PV paravertebral 
the injection was performed and the interval from injection until commencing of dissection was limited to 20 min. In our study, a single, expert in US guided RA performed all injections in order to limit variation in spread attributable to differences in operator performance.

There are some limitations to our study. The total number of dissections performed was small $(n=10)$ and limits the generalizability of our findings to patients of different size and body habitus. Another limitation was the lack of normal ventilatory movement. The intercostal spaces and erector spinae muscles move in synchrony with ventilation. As these movements occur, spaces and potential openings may result to permit or even propel injectate. The number of injections per volume also varied and was not uniform. Given the absence of dye spread following $10 \mathrm{ml}$ injection to any clinically relevant areas, a decision was made early in the course of the study to focus on clinically relevant volumes $(20 \mathrm{ml}$ and $30 \mathrm{ml})$. This enabled more complete analysis of spread patterns for those volumes and represented better use of the cadaver specimens.

\section{Conclusion}

Based on these findings, we conclude that the chest wall analgesia associated with RB is affected through blockade of neural structures at more than one site. In particular, injection of a greater volume appears to increase the likelihood of spread to the PVS. US guidance does not offer a means by which spread to the PVS can be reliably detected evidenced by poor interobserver reliability.

In view of the much lesser likelihood of pneumothorax with RB, it may offer a safe effective option for anaesthesiologists who are not expert in performance of PVB. Further work is justified to understand its site and mechanism of action. This could inform development of a safe and consistently effective modification of the block.

\section{Conflict of interest: none}

\section{References}

1. Wu J, Buggy D, Fleischmann E, et al. Thoracic paravertebral regional anesthesia improves analgesia after breast cancer surgery: a randomized controlled multicentre clinical trial. J Clin Anesth 2015;62:241-251.
2. Andreae MH, Andreae DA. Regional anaesthesia to prevent chronic pain after surgery: a Cochrane systematic review and meta-analysis. Br J Anaesth 2013;111:711-720.

3. Ziegler DW, Agarwal NN. The morbidity and mortality of rib fractures. J Trauma Acute Care Surg 1994;37:975-979.

4. Sirmali M, Türüt H, Topçu S, et al. A comprehensive analysis of traumatic rib fractures: morbidity, mortality and management. Eur J Cardiothorac Surg 2003;24:133-138.

5. Galvagno Jr SM, Smith CE, Varon AJ, et al. Pain management for blunt thoracic trauma: A joint practice management guideline from the Eastern Association for the Surgery of Trauma and Trauma Anesthesiology Society. J Trauma Acute Care Surg 2016;81:936-951.

6. Mohta M, Verma P, Saxena AK, et al. Prospective, randomized comparison of continuous thoracic epidural and thoracic paravertebral infusion in patients with unilateral multiple fractured ribs - a pilot study. J Trauma Acute Care Surg 2009;66:1096-1101.

7. Yeung JH, Gates S, Naidu BV, et al. Paravertebral block versus thoracic epidural for patients undergoing thoracotomy. Cochrane Libr 2016.

8. Naja Z, Lönnqvist PA. Somatic paravertebral nerve blockade incidence of failed block and complications. Anaesthesia 2001;56:1181-1201.

9. Schnabel A, Reichl SU, Kranke P, et al. Efficacy and safety of paravertebral blocks in breast surgery: a meta-analysis of randomized controlled trials. Br J Anaesth 2010;105:842852.

10. Pace MM, Sharma B, Anderson-Dam J, et al. Ultrasoundguided thoracic paravertebral blockade: a retrospective study of the incidence of complications. Anesth Analg 2016;122:1186-1191.

11. Krediet AC, Moayeri N, van Geffen GJ, et al. Different Approaches to Ultrasound-guided Thoracic Paravertebral Block: An Illustrated Review. Anesthesiology 2015;123(:459-74.

12. Pfeiffer G, Oppitz N, Schone S, et al. Analgesia of the axilla using a paravertebral catheter in the lamina technique (German). Anaesthesist 2006;55:423-427.

13. Jüttner T, Werdehausen R, Hermanns $H$, et al. The paravertebral lamina technique: a new regional anesthesia approach for breast surgery. J Clin Anesth 2011;23:443-450.

14. Voscopoulos C, Palaniappan D, Zeballos J, et al. The ultrasound-guided retrolaminar block. J Clin Anesth. 2013;60:888-895.

15. Murouchi T, Yamakage M. Retrolaminar block: analgesic efficacy and safety evaluation. J Anesth 2016;30:10031007.

16. Sabouri AS, Crawford L, Bick SK, et al. Is a retrolaminar approach to the thoracic paravertebral space possible? A human cadaveric study. Reg Anesth Pain Med 2018;43:864868. 